\title{
A Cosynthesis Algorithm for Application Specific Processors with Heterogeneous Datapaths
}

\author{
Yuichiro Miyaoka $^{\dagger} \quad$ Nozomu Togawa $^{\dagger \dagger} \quad$ Masao Yanagisawa $^{\ddagger} \quad$ Tatsuo Ohtsuki $^{\ddagger}$ \\ Waseda University The University of Kitakyushu Waseda University Waseda University \\ miyaoka@ohtsuki.comm.waseda.ac.jp togawa@env.kitakyu-u.ac.jp yanagi@yanagi.comm.waseda.ac.jp to@ohtsuki.comm.waseda.ac.jp \\ † Dept. of Electronics, Information and Communcation Engineering, Waseda University \\ 3-4-1, Okubo, Shinjuku, Tokyo, 169-8555, Japan, Tel: +81-3-5286-3396, Fax: +81-3-3203-9184 \\ i† Dept. of Computer and Media Sciences, The University of Kitakyushu \\ 1-1, Hibikino, Wakamatsu, Kitakyushu, 808-0135, Japan, Tel: +81-93-695-3264, Fax: +81-93-695-3368 \\ $\$$ Dept. of Computer Science, Waseda University \\ 3-4-1, Okubo, Shinjuku, Tokyo, 169-8555, Japan, Tel: +81-3-5286-\{3392,3387\}, Fax: +81-3-\{3204-4875, 3203-9184 \}
}

\begin{abstract}
This paper proposes a hardware/software cosynthesis algorithm for processors with heterogeneous registers. Given a CDFG corresponding to an application program and a timing constraint, the algorithm generates a processor configuration minimizing area of the processor and an assembly code on the processor. First, the algorithm configures a datapath which can execute several DFG nodes with data dependency at one cycle. The datapath can execute the application program at the least number of cycles. The branch and bound algorithm is applied and all the number of functional units and memory banks are tried. For an assumed number of functional units and memory banks, an appropriate number of heterogeneous registers and connections to functional units and registers are explored. The experimental results show effectiveness and efficiency of the algorithm.
\end{abstract}

\section{INTRODUCTION}

General DSPs such as TMS320C2x[14], DSP56300[12], DSP 16xx[10], ADSP-21 $\times x$ [3], and [9] have heterogeneous datapaths. Heterogeneous registers (accumulate registers, for example) can have flexible bit width, while general purpose registers must have single bit width. Heterogeneous registers can satisfy application requirements with less hardware costs. Sophisticated heterogeneous datapaths including heterogeneous registers can execute application programs fast. Therefore, processors with heterogeneous datapaths can have small costs and achieve high performance.

For processors with heterogeneous datapaths, code optimization or generation is a struggled problem, since, for example, it must be considered which of heterogeneous registers a variable is bound to. Several retargetable compilers to processors with heterogeneous datapaths have been reported as in $[5,6,7,11,17,18]$. These retargetable compilers, however, cannot always make a sufficient application code, since datapath configuration of the target processor is not always suitable for a given application program. We think that an application specific processor should be synthesized, especially for a processor with a heterogeneous datapath. A Hardware/software codesign method can be effectively applied to processors with a heterogeneous datapaths. Several researches on a hardware/software codesign for microprocessors have been reported as in $[1,2,4,13,15]$. However, they does not focus on heterogeneous datapaths. In [8] a hardware/software codesign environment have been proposed. In [8], given an application program and datapath configuration, the codesign environment generates a processor hardware description and the object code on the processor. However, another datapath configuration must be manually designed when estimated area or the execution time of a given application program on a processor with a datapath configuration is insufficient. At the design of a processor with a heterogeneous datapath, it is difficult to find a performance bottleneck and to design another appropriate processor datapath. Exploring datapath configuration and compiling an application program must be close each other.

Therefore we propose a hardware/software cosynthesis algorithm for processors with heterogeneous datapaths. Given a CDFG corresponding to an application program and a timing constraint, the algorithm generates a processor configuration minimizing area of the processor and an assembly code on the processor. First, the algorithm configures a datapath which can execute several DFG nodes with data dependency at one cycle. The datapath can execute the application program at the least number of cycles. The branch and bound algorithm is applied and all the number of functional units and memory banks are tried. For an assumed number of functional units and memory banks, an appropriate number of heterogeneous registers and connections to functional units and registers are explored.

This paper is organized as follows: Section II defines a processor architecture. Section III proposes a hardware/software cosynthesis algorithm for processors with heterogeneous datapaths. Section IV shows experimental results. Section $V$ gives concluding remarks.

\section{ARCHITECTURE MODEL}

This section defines a processor architecture model for our synthesis algorithm. Our VLIW type processor has the 3 stage pipelines composed of IF, ID, and EXE stages. Immediate values are decoded on the ID stage and written in the ID/EXE pipeline registers. The processor can have one or two data memory banks. The data bus width of data memory is fixed to bit width of processor basic bit width $b_{\text {basic }}$. Besides the number of data memory banks, configuration of general purpose registers, heterogeneous registers, and functional units can be changed. Figure 1 shows our processor model. 


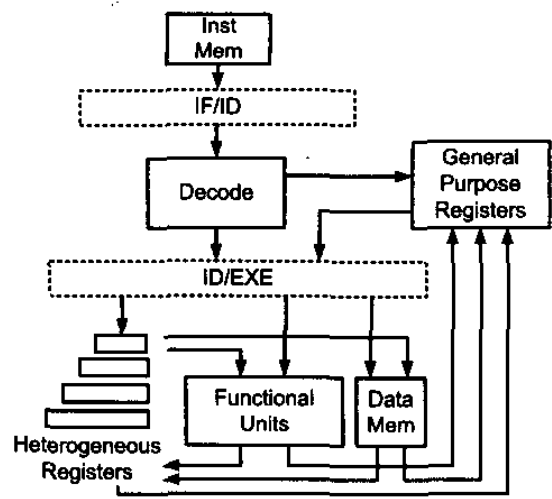

Fig. 1. Our pipeline architecture.

Bit width of general purpose registers is $b_{b a s i c}$. In case that data of a general purpose register is read, the data is read at the ID stage and saved in the ID/EXE pipeline registers. In case that data is written in a general purpose register, data is generated at the EXE stage and written back to the register. The number $n_{g}$ of general registers is given in advance.

Data of heterogeneous registers are read and written at the EXE stage. Data read from a heterogeneous register is (a) used for an input of a functional unit, (b) written back to a general purpose register, (c) written back to another heterogeneous register, or (d) written to data memory. To convert bit width of data in a heterogeneous register to bit width required for (a)(d), a heterogeneous register can have a bit extended/extracted unit. (e) Output data of a functional unit, (f) data in a general purpose register, $(\mathrm{g})$ data in another heterogeneous register, or (h) data from data memory is written to a heterogeneous register. The number of heterogeneous registers and bit width of each heterogeneous register can be changed. Which of (a)-(h) connections each register has and whether each register has a bit extended/extracted unit can be changed.

Functional units are such as ALUs, adding units, multiplying units, shift operation units, and bit extended/extracted units. For the same operation type, a functional unit can have different bit width of inputs and outputs. A processor has several functional units in a functional unit library $F U=\left\{f_{1}, f_{2}, \ldots f_{u}\right\}$. By connecting more than one functional units, a processor can have a datapath which can execute a operation and another operation continually. For example, by connecting an output port of a multiplying unit to an input port of an adding unit, multiplying and adding operation can be executed at one cycle.

We define minimum instructions to perform as a general processor. A processor must have a datapath which can execute (in one or several cycles) adding operation, logical and/or/xor operation, shift operation, and comparing operation for two variables $a$ and $b$ in the data memory $m_{0} m_{0}$, and write their operation results to $m_{e} m_{0}$. A processor must have a nonconditional branch operation and a branch-on-equal operation for $a$ and $b$.

$n_{g}, n_{r}$ and $n_{w}$, and $n_{h}$ denote the number of general purpose registers, reading ports and writing ports of general purpose registers, and heterogeneous registers respectively. $n_{f}$ denotes the number of functional units in a processor. $n_{m} \in\{1,2\}$

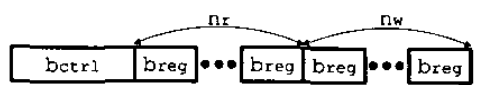

Fig. 2. Instruction format.

denotes the number of data memory banks and $s_{m e m_{i}}$ denotes the number of signals which can be written to data memory $i$. Let $s_{g}$ be the number of signals which can be written back to general purpose registers and $s_{h}^{i}$ be the number of signals which can be written back to a heterogeneous register $R_{h}^{i}$. Assume that a functional unit $f_{i}$ should have $n_{f_{i}}$ input ports and that the number of signals which connect a $j\left(0 \leq j<n_{f_{i}}\right)$-th input of a functional unit should be $s_{f_{i}}^{j}$. Then an instruction set is composed of, at most,

$$
n_{\text {inst }}=\prod_{i=0}^{n_{m}-1} s_{m e m_{i}} \times s_{g} \times n_{w} \times \prod_{i=0}^{n_{h}-1} s_{h}^{i} \times \prod_{i=0}^{n_{f}-1} \prod_{j=0}^{n_{f_{i}}-1} s_{f_{i}}^{j}
$$

instructions. Only the instructions which are required in order to execute a given application program and minimum instructions as mentioned above are synthesized. Bit length for an instruction code is $b_{c t r l} \leq\left\lceil\log n_{\text {inst }}\right\rceil$ and bit length for specifying general purpose registers is $\left(n_{r}+n_{w}\right) \times b_{r e g}$, where $b_{r e g}$ is bit length to specify a general register and $b_{\text {reg }}=\left\lceil\log n_{g}\right\rceil$ (Fig. 2).

We estimate processor area by adding up area of (i) ID/EXE pipeline registers, (ii) general purpose registers, (iii) heterogeneous registers, (iv) functional units, and (v) multiplexers (for writing to general purpose registers, heterogeneous registers, and data memory banks, and input of functional units). Let $a_{r e g}$ and $a_{m u x}$ be area of a single bit register and a 21 multiplexer respectively. Area $A_{\text {pipe }}$ of (i) is estimated as $A_{\text {pipe }}=a_{\text {reg }} \times\left(b_{\text {ctrl }}+n_{r} \times b_{\text {basic }}+n_{w} \times b_{\text {reg }}\right)$. Area $A_{g}$ is estimated as $A_{g}=a_{\text {reg }} \times n_{g} \times b_{\text {basic }}+a_{\text {mux }} \times\left(n_{r} \times b_{\text {basic }} \times\right.$ $\left.\left(n_{g}-1\right)+n_{w} \times b_{\text {basic }} \times n_{g}\right)$. Area $A_{h}$ of (iii) is estimated as $A_{h}=a_{r e g} \times \sum_{i=0}^{n_{h}-1} b_{h}^{i}$, where $b_{h}^{i}$ is bit width of $R_{h}^{i}$. Area $A_{F}$ of (iv) is estimated as $A_{F}=\sum_{f u \in F} a_{f u}$. Finally, area $A_{m u x}$ of (v) is estimated as $A_{m u x}=a_{m u x} \times \sum_{p \in \text { allmux }}\left(s_{p}-1\right) \times b_{p}$, where $s_{p}$ is the number of signals which can be input to a multiplexer $p$ and $b_{p}$ is bit width of the multiplexer $p$.

The clock period of a processor is estimated as critical path delay at the EXE stage, which is composed of delay of multiplexers, functional units, and writing back to registers. We assume that $d_{m u x}$ should be delay of a 2-1 multiplexer and we estimate delay of an $s_{p}-1$ multiplexer as $d_{m u x} \times\left\lceil\log s_{p}\right\rceil$.

\section{A HARDWARE/SOFTWARE COSYNTHESIS ALGORITHM} FOR PROCESSORS WITH HETEROGENEOUS DATAPATHS

This section proposes an algorithm to synthesize a processor with a heterogeneous datapath.

\section{A. Problem definition}

A control flow graph (CFG) $G_{c}=\left(V_{c}, E_{c}\right)$ is defined as a graph representing control flow in a function. A CFG has no input and output edges. A basic block is a node of a CFG and has a data flow graph (DFG). A basic block has no branches and joins except for its starting and ending. A DFG $G_{d}=\left(V_{d}, E_{d}\right)$ is defined as a graph representing data flow. An ending node of a DFG may be connected to a starting node in another DFG. A DFG is a set of operations from a branch or 


\section{$3 A-3$}

join to a next branch or join in an application. A node $v \in V_{d}$ is associated with an operation in a functional unit $f \in F U$ or a memory access operation. If the execution of a DFG node $v_{1}$ has data dependency to another DFG node $v_{2}$, a DFG has an edge $\left(v_{1}, v_{2}\right)$.

$T_{a p p}$, the execution time of an application program, is calculated by a product of the number of total clock cycles to execute the CDFG and a clock period of the processor. Processor configuration includes the number of data memory banks, the number of heterogeneous registers, bit width of each heterogeneous register, the types and number of functional units, and the connections among data memory, general purpose registers, heterogeneous registers, and functional units.

Definition III.1 (Processor synthesis problem) A processor synthesis problem is to determine processor configuration given a CDFG and a timing constraint in order to minimize processor area while the execution time of a given application program satisfies a timing constraint $T_{\max }$, that is, $T_{a p p} \leq$ $T_{\max }$.

\section{B. Proposed algorithm}

The proposed algorithm is composed of two phases. One determines initial processor configuration and the other explores processor configuration. Initial processor configuration has a sufficient number of functional units and heterogeneous registers, and can execute the application program in a small number of cycles, while processor area of the configuration is large. The branch and bound algorithm is applied and all the number of functional units and memory banks are tried. For an assumed number of functional units and memory banks, an appropriate number of heterogeneous registers and connections to functional units and registers are explored. In this way, we can obtain optimized processor configuration in a short time.

\section{B.1 Initial processor configuration}

To configure initial processor configuration, all the DFGs in a CFG are scheduled in the following method. To schedule a DFG $G_{d}=\left(V_{d}, E_{d}\right)$ associated with a CFG node $v_{c} \in V_{c}$ is to determine a pair $(v, s t)$ for all the nodes $v \in V_{d}, s t \in$ $\{1,2, \ldots\}$, that is, to construct $S_{v_{c}}=\left\{\left.(v, s t)\right|^{\forall} v \in V_{d}\right\}$. A node $v \in V_{d}$ is a node executed by a functional unit (called a functional node) or a node accessing data memory (called $a$ memory accessing node). A set of all the functional nodes in $V_{d}$ is denoted as $V_{f}$. A set of all the memory accessing node in $V_{d}$ is denoted as $V_{m}$, where $V_{f}$ and $V_{m}$ satisfy $V_{d}=V_{f} U$ $V_{m}, V_{f} \cap V_{m}=\phi$. Our target processor can execute several functional nodes at one cycle which have data dependency to one another by connecting an output of a functional unit to an input of another functional unit. However, a processor cannot execute a node to load data from memory and a node to operate the loaded data at the same cycle and cannot execute a node to store data to memory and a node to operate the stored data at the same cycle. Therefore, if an edge $\left(v_{i}, v_{j}\right)$ is included in $E_{d}$ and $v_{i}$ and $v_{j} \in V_{d}$ are assigned to a step $s t_{i}$ and $s t_{j}$ respectively, $s t_{i} \leq s t_{j}$ in $v_{i}, v_{j} \in V_{f}$ and $s t_{i}<s t_{j}$ in $v_{i} \in V_{m}$ or $v_{j} \in V_{m}$. Figure 3 shows the step assigning rule. DFG nodes are assigned to steps in a topological order. To assign a functional node and memory accessing node to a step $s t$ is realized by assigning all the functional nodes which can be assigned to $s t$ and memory accessing nodes satisfying resource constraints. Figure 4 shows this algorithm.

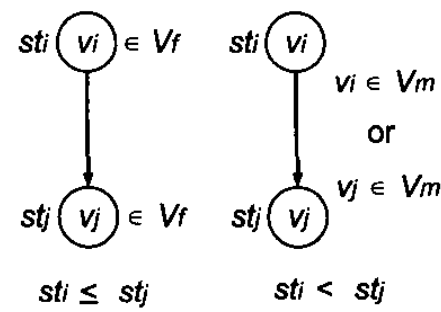

(a)

(b)

Fig. 3. A step assigning rule. (a) In case a predecessor and successor node of an edge is in $V_{f}$, (b) a predecessor or successor node is in $V_{m}$.

Inputs A CFG $G_{\mathrm{c}}=\left(V_{c}, E_{\mathrm{c}}\right)$ and DFG $G_{\mathrm{d}}=\left(V_{\mathrm{d}}, E_{\mathrm{d}}\right)$.

Outputs A scheduling result $\left\{\left.S_{v_{c}}\right|^{\forall} v_{c} \in V_{c}\right\}$.

Step 1. Select a CFG node $v_{c} \in V_{c}$. Execute $s t \leftarrow 1$ and $S_{v_{c}} \leftarrow \emptyset$.

Step 2. Select $v \in V_{d}$ in $G_{d}=\left(V_{d}, E_{d}\right)$ corresponding to $v_{c}$ in a topological order.

Step 2.1. For $v \in V_{f}$, assign $v$ to $s t$ if there are no $v^{\prime}$ where $v^{\prime} \in V_{m}$ and $\left(v^{\prime}, v\right) \in E_{d}$. Execute $S_{v_{c}} \leftarrow S_{v_{c}} \cup(v, s t)$.

Step 2.2. For $v \in V_{m}$, assign $v$ to $s t$ if a memory resource constraint is satisfied and a step $s t^{\prime}$ assigned $v^{\prime}$ where $\left(v^{\prime}, v\right) \in E_{d}$ is less than st. Execute $S_{v_{c}} \leftarrow S_{v_{c}} \cup(v, s t)$

Step 3. Go to Step 4 if every node in $V_{d}$ are assigned to a step. Otherwise, update $s t \leftarrow s t+1$ and go to Step 2.

Step 4. $S_{v_{c}}$ has been obtained. Select another CFG node $v_{c}{ }^{\prime}$ and go to Step 2. Finish the algorithm when all the CFG nodes have been scheduled.

Fig. 4. Initial scheduling algorithm.

Based on an initial scheduling result, an initial processor configuration is configured. If a predecessor node and a successor node of an edge are assigned to different steps, the edge is assigned to a register. The lifetime of each variable is analyzed in advance.

First, we decide the number of functional units. Let $V_{v_{c}, s t}$ be a set of nodes assigned to a step st in a node $v_{c} \in V_{c} . f u(v)$ denotes a functional unit or data memory corresponding to $v \in$ $V_{d}$. The number of a functional unit $f_{i}$ to execute $V_{v_{c}, s t}$ in a cycle is $n_{f_{i}}\left(v_{c}, s t\right)=\left|\left\{v \in V_{v_{c}, s t} \mid f u(v)=f_{i}\right\}\right|$. Therefore, the number of a functional unit $f_{i}$ which a processor has is,

$$
n_{f_{i}}=\max _{\forall v_{c}, \forall s t}\left\{n_{f_{i}}\left(v_{c}, s t\right)\right\}
$$

Similarly, the number of data memory banks is determined to be one or two.

Now, we propose an algorithm to configure the connections among functional units, data memory banks, and registers. We define a processor configuration graph in order to represent the connections. A processor configuration graph denotes $G_{p r}=\left(V_{p r}, E_{p r}\right)$. A node in $V_{p r}$ correspond to a pipeline register, a heterogeneous register, a functional unit, or data memory. A processor configuration graph has an edge $\left(v_{1}, v_{2}\right)$ if there is a connection between hardware corresponding to $v_{1}$ and one corresponding to $v_{2}$. The number of nodes corresponding to functional units or data memory can be determined as mentioned above. Thus, there are $n_{f_{i}}$ functional units associated with $f_{i}$ in $v_{p r}$. There are one or two nodes associated with data memory banks. Corresponding to reading 


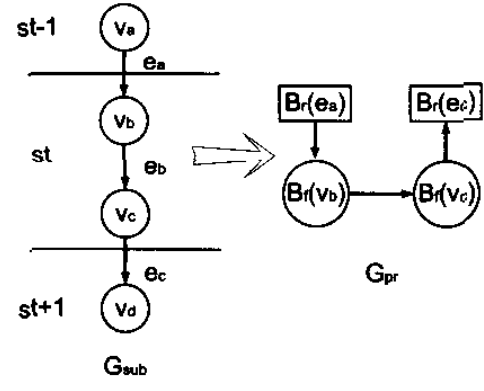

Fig. 5. An example of $G_{p r}$ satisfying configuring conditions based on $G_{s u b}$.

and writing general purpose registers, nodes associated with pipeline registers must be included in $V_{p r}$.

First, to perform as a general processor, our algorithm adds a node $v$ associated with an ALU to $V_{p r}$ and edges between $v$ and nodes associated with pipeline registers.

The number of nodes associated with heterogeneous registers and a set $E_{p r}$ of edges are decided by applying the following algorithm to all the steps of all the basic blocks and updating $G_{p r}=\left(V_{p r}, E_{p r}\right)$.

Let $E_{s u b}$ be a set of edges where $\left(v_{1}, v_{2}\right) \in E_{d}, v_{1} \in V_{v_{c}, s t}$ or $v_{2} \in V_{v_{c}, s t}$. A subgraph $G_{s u b}=\left(V_{s u b}, E_{s u b}\right)$ of $G_{p r}$ can be constructed, where $V_{s u b}$ is a set of all the nodes which are $v_{1}$ or $v_{2}$ of $\left(v_{1}, v_{2}\right) \in E_{s u b}$. Configuring conditions for $G_{p r}$ in a step st is defined as follows.

1. $v_{d} \in V_{v_{c}, s t}$ is associated with $v_{p r} \in V_{p r}$. A mapping function is denoted as $B_{f}: V_{v_{c}, s t} \mapsto V_{p r}$. If $v_{p r}=$ $B_{f}\left(v_{d}\right), v_{p r}$ is corresponding to a hardware $f u\left(v_{d}\right)$. If $v_{1}, v_{2} \in V_{v_{c}, s t}, B_{f}\left(v_{1}\right) \neq B_{f}\left(v_{2}\right)$.

2. If $v_{1}, v_{2} \in V_{v_{c}, s t}$ for $e=\left(v_{1}, v_{2}\right) \in E_{s u b}$, $\left(B_{f}\left(v_{1}\right), B_{f}\left(v_{2}\right)\right)$ must be included in $E_{p r}$.

3. Let $E_{r}=\left\{\left(v_{s}, v_{t}\right) \in E_{s u b}\right\}$ be a set of edges where $v_{s} \notin V_{v_{c}, s t}$ or $v_{t} \notin V_{v_{c}, s t} . \quad e_{r} \in E_{r}$ is associated with $v_{p r} \in V_{p r}$. A mapping function is denoted as $B_{r}: E_{r} \mapsto V_{p r}$. If $v_{p r}=B_{r}\left(e_{r}\right), v_{p r}$ is corresponding to a heterogeneous register between $B_{f}\left(v_{s}\right)$ and $B_{f}\left(v_{t}\right)$. For $e_{1}=\left(v_{i}, v_{j}\right), e_{2}=\left(v_{k}, v_{l}\right) \in E_{s u b}, B_{r}\left(e_{1}\right)=B_{r}\left(e_{2}\right)$ if $v_{i}=v_{k}$ and $B_{r}\left(e_{1}\right) \neq B_{r}\left(e_{2}\right)$ if $v_{i} \neq v_{k}$.

4. If $v_{1} \in V_{v_{c}, s t}$ and $v_{2} \notin V_{v_{c}, s t}$ for $e=\left(v_{1}, v_{2}\right) \in E_{s u b}$, $\left(B_{f}\left(v_{1}\right), B_{r}(e)\right)$ must be included in $E_{p r}$. Similarly, if $v_{1} \notin V_{v_{c}, s t}$ and $v_{2} \in V_{v_{c}, s t}$ for $e=\left(v_{1}, v_{2}\right) \in E_{s u b}$, $\left(B_{\tau}(e), B_{f}\left(v_{2}\right)\right)$ must be included in $E_{p r}$.

Figure 5 shows an example of $G_{s u b}$ and a graph satisfying configuring conditions for $G_{p r}$ in a step $s t$.

A mapping function $B_{f}$ which corresponds $V_{v_{c}, s t}$ to $V_{p r}$ is decided so that the configuring condition 1 for $G_{p r}$ is satisfied. Then, $G_{p r}$ is updated as follows so that configuring conditions $2-4$ for $G_{p r}$ are satisfied.

1. If $\left(B_{f}\left(v_{1}\right), B_{f}\left(v_{2}\right)\right)$ is in $E_{p r}$ for $\left(v_{1}, v_{2}\right) \in E_{s u b}$ where $v_{1}$ and $v_{2}$ are in $V_{v_{c}, s t}, E_{p r}$ is not updated. If $\left(B_{f}\left(v_{1}\right), B_{f}\left(v_{2}\right)\right) \notin E_{p r},\left(B_{f}\left(v_{1}\right), B_{f}\left(v_{2}\right)\right)$ is added to $E_{p r}$.
Inputs A scheduling result $\left\{\left.S_{v_{c}}\right|^{\forall} v_{c} \in V_{c}\right\}$ and a set of subgraph $G_{s u b}=$ $\left(V_{s u b}, E_{s u b}\right)$.

Outputs A processor configuration graph $G_{p r}=\left(V_{p r}, E_{p r}\right)$.

Step 1. Calculate the number $n_{f_{i}}$ of $f_{i}$ as

$$
n_{f_{i}}=\max _{\forall v_{c}, \forall s t}\left\{n_{f_{i}}\left(v_{c}, s t\right)\right\}
$$

Similarly, the number of data memory banks is determined to be one or two.

Step 2. Include nodes associated with functional units, data memory banks, and reading and writing general purpose registers. The numbers of nodes associated with functional units and data memory banks are decided at Step 1. $E_{p r} \leftarrow \phi$.

Step 3. Add a node $v$ associated with an ALU to $V_{p r}$ and edges between $v$ and nodes associated with pipeline registers to $E_{p r}$.

Step 4. Pick up a CFG node $v_{\mathrm{c}} . s t \leftarrow 0$.

Step 5. Decide a mapping function $B_{f}$ which associates functional nodes in $G_{s u b}$ at $s t$ to the nodes in $G_{p r}$. Try to update $G_{p r}$ and calculate the $G_{p r}$ cost.

Step 5.1. If $\left(B_{f}\left(v_{1}\right), B_{f}\left(v_{2}\right)\right)$ is in $E_{p r}$ for $\left(v_{1}, v_{2}\right) \in E_{s u b}$ where $v_{1}$ and $v_{2}$ are in $V_{v_{c}, s t}, E_{p r}$ is not updated. If $\left(B_{f}\left(v_{1}\right), B_{f}\left(v_{2}\right)\right) \notin E_{p r}, E_{p r}-E_{p r} \cup\left(B_{f}\left(v_{1}\right), B_{f}\left(v_{2}\right)\right)$.

Step 5.2. Let us focus on an edge $e=\left(v_{1}, v_{2}\right) \in E_{r}$ where $v_{1} \in$ $V_{v_{c}, s t}$ and $v_{2} \notin V_{v_{c}, a t}$. Let $v_{\text {reg }}$ be a node which is associated with a heterogeneous register connected to an outgone edge of $B_{f}\left(v_{1}\right)$ and does not have st in lifetime. Update a mapping function $B_{r}$ so that $B_{r}(e)=v_{r e g}$. If there does not exist such $v_{r e g}, V_{p r} \leftarrow V_{p r} \cup v_{r e g}$ and $B_{r}(e)=v_{r e g}$ is added to $B_{r}$.

Step 5.3. For $e=\left(v_{1}, v_{2}\right) \in E_{r}$ where $v_{1} \in V_{v_{c}, s t}$ and $v_{2} \notin V_{v_{c}, s t}, B_{r}(e)$ has been determined at an iteration by $s t-1$. If $\left(B_{r}(e), B_{f}\left(v_{2}\right)\right)$ is not in $E_{p r}, E_{p r} \leftarrow E_{p r} \cup$ $\left(B_{r}(e), B_{f}\left(v_{2}\right)\right)$.

Step 6. Select $B_{f}$ which has the minimum $G_{p r}$ cost and update $G_{p r}$.

Step 7. $s t \rightarrow s t+1$ and go to Step 5. if the steps for all the $s t$ are tried, go to Step 4. Finish the algorithm if all the CFG nodes are tried.

Fig. 6. A processor configuration algorithm.

2. Let us focus on an edge $e=\left(v_{1}, v_{2}\right) \in E_{r}$ where $v_{1} \in V_{v_{c}, s t}$ and $v_{2} \notin V_{v_{c}, s t}$. Let $v_{r e g}$ be a node which is associated with a heterogeneous register connected to an outgone edge of $B_{f}\left(v_{1}\right)$ and does not have $s t$ in lifetime. Update a mapping function $B_{r}$ so that $B_{r}(e)=v_{\text {reg }}$. If there does not exist such $v_{r e g}$, a new $v_{r e g}$ is included in $V_{p r}$ and $B_{r}(e)=v_{r e g}$ is added to $B_{r}$.

3. For $e=\left(v_{1}, v_{2}\right) \in E_{r}$ where $v_{1} \in V_{v_{c}, s t}$ and $v_{2} \notin V_{v_{c}, s t}$, $B_{r}(e)$ has been determined at an iteration by $s t-1$. If $\left(B_{r}(e), B_{f}\left(v_{2}\right)\right)$ is not in $E_{p r},\left(B_{r}(e), B_{f}\left(v_{2}\right)\right)$ is included in $E_{p r}$.

The algorithm selects $B_{f}$ which has the minimum $G_{p r}$ cost and updates $G_{p r}$, where $G_{p r}$ cost is defined by estimating processor area as mentioned in Sect. II. The processor configuration algorithm is shown in Fig. 6.

An initial processor configuration is obtained by applying the processor configuration algorithm to an initial scheduling result. An initial processor configuration has the minimum number of cycles executing the application program. Based on a model in Sect. II, area and a clock period of the processor is estimated. 
Inputs A processor configuration graph $G_{p r}=\left(V_{p r}, E_{p r}\right)$ obtained by means of applying the processor configuration algorithm.

outputs A updated processor configuration graph $G_{p r}{ }^{\prime}=\left(V_{p r}{ }^{\prime}, E_{p r}{ }^{\prime}\right)$.

Step. 1 Try to reduce for one of all the registers as following two reduction methods. Calculate processor area and the execution time of the application program.

1. Focus on two heterogeneous registers which have same bit width and have no overlap in their lifetime. If a register $v_{2}$ is, Update all the edges which have $v_{2}$ in either side of edges by replacing $v_{2}$ to $v_{1} . V_{p r} \leftarrow V_{p r} \backslash v_{2}$ and Update $E_{p r}$.

2. Reduce a heterogeneous register which has the same bit width as general purpose registers and Save a content of the heterogeneous register in a general register. Update all the edges which have the heterogeneous register $v$ in either side of edges by replacing $v_{2}$ to a pipeline register. $V_{p r} \leftarrow V_{p r} \backslash v$ and Update $E_{p r}$.

Step 2. Actually update a processor configuration which has the minimum area under the timing constraint of all the candidates. New $G_{p r}$ is obtained. If there are more than one such processor configurations, select the candidate in which the number of edges in $E_{p r}$ including the node associated with the heterogeneous register is the most.

Step 3. Finish if there are no registers that can be reduced in Step 1 or all the candidates do not satisfy a timing constraint.

Fig. 7. An algorithm to reduce heterogeneous registers.

\section{B.2 Exploring a processor configuration}

Based on an initial processor configuration, the maximum numbers of functional units and data memory banks are determined. We apply a branch and bound method which subproblems are constructed by branching about the number of functional units and data memory banks. When the numbers of functional units and data memory banks are determined, we have only to configure registers. Therefore we optimize processor configuration in a short time. We solve the sub-problem as follows. First we schedule a CDFG under a constraint of the numbers of functional units and data memory banks. Figure 4 can be easily applied to it by modifying that assigned nodes are restricted by resource constraints. The processor configuring algorithm is applied to the scheduling result. While a timing constraint is satisfied, a heterogeneous register reduction algorithm shown in Fig. 7 is applied.

\section{EXPERIMENTAL RESULTS}

We have implemented the proposed algorithm in $\mathrm{C}++$ on Sun Ultra Sparc $3750 \mathrm{MHz}$. We use gcc 2.95 .3 as a compiler. To estimate area and delay of hardware, we use VDEC libraries (CMOS and $0.35 \mu \mathrm{m}$ technology) ${ }^{1}$. We use area and delay in Table I for estimation of functional units. We assume that area and delay of 2-1 multiplexer are $a_{m u x}=167\left[\mu \mathrm{m}^{2}\right]$ and $d_{m u x}=0.23$ [ns], respectively and that area and writing delay of a single bit register are $a_{\text {reg }}=383\left[\mu \mathrm{m}^{2}\right]$ and $d_{\text {reg }}=0.40$ [ns], respectively. We assume that basic bit width of a processor is 16 bit.

The algorithm has been applied to an FIR filter and a DCT in which basic bit width of variables is 16 bit and 32 bit multiplying and accumulate operation are used. The results are shown in Table II. For different timing constraints, different processor configuration can be obtained. In order to compare

\footnotetext{
'The libraries in this study have been developed in the chip fabrication program of VLSI Design and Education Center (VDEC), the University of Tokyo with the collaboration by Hitachi Ltd. and Dai Nippon Printing Corporation.
}

TABLE I

FUNCTIONAL UNITS

\begin{tabular}{|crr|}
\hline Unit & Area $\left[\mu \mathrm{m}^{2}\right]$ & Delay [ns] \\
\hline ADD16 & 25,259 & 1.44 \\
ALU16 & 78,915 & 2.82 \\
MUL16 & 356,948 & 5.71 \\
ADD32 & 41,963 & 2.49 \\
ALU32 & 151,194 & 3.25 \\
\hline
\end{tabular}

our results with existing systems, we have picked up two processor synthesis systems (which are referred to as [15] and [16] in References. [15] synthesizes a simple processor with a homogeneous datapath. [16] synthesizes a processor which has two types of register files and a homogeneous datapath. Comparison results have been shown in Table III. It shows that our system can synthesize processors with less area than existing systems which synthesize processors with homogeneous datapaths. When the timing constraint of $60 \mu \mathrm{s}$ is given, System 1 and 2 cannot output a processor configuration meeting the timing constraint.It is because processors synthesized by system 1 and 2 cannot have chains among the functional units and no processors satisfy the timing constraint. Our proposed algorithm synthesizes a processor with less area compared with System 1 because of the following reason: In a processor synthesized by system 1 , all the registers and functional units must have bit width of 32 bits. In a processor synthesized by the proposed algorithm, however, registers and functional units can have flexible bit width. The processor can have both 16 bits and 32 bits resources. Our proposed algorithm synthesizes a processor with less area compared with System 2 because of the following reason: A processor synthesized by system 2 can have 32-bit and 16-bit registers and functional units. Since the synthesized processor, however, has a homogeneous datapath, it must have connections and multiplexers between all the registers and all the functional units. A processor synthesized by the proposed algorithm can have only connections and multiplexers required in order to execute a given application program. Therefore we can synthesize a processor with less area.

\section{CONCLUSION}

In this paper, we proposed a hardware/software cosynthesis algorithm for processors with heterogeneous datapaths. In the future, we will incorporate SIMD functional units into the processor model and establish the algorithm to optimize the processor configuration.

\section{REFERENCES}

[1] $\mathrm{H}$. Akaboshi, and H. Yasuura, COACH: A computer aided design tool for computer architectures, IEICE Transactions on Fundamentals of Electronics, Communications and Computer Sciences, vol. E76-A, no. 10, pp. 1760-1769, 1993.

[2] A. Alomary, T. Nakata, Y. Honma, M. Imai, and N. Hikichi, “An ASIP instruction set optimization algorithm with functional module sharing constraint," in Proceeding of 1993 IEEE/ACM International Conference on Comuputer-Aided Design, pp. 526-532, 1993.

[3] Analog Devices, ADSP-2100 Family User's Manual, 1995.

[4] N. N. Bình, M. Imai, A. Shiomi, and N. Hikichi, "A hardware/software partitioning algorithm for designing pipelined ASIPs with least gate count," in Proceedings of 33rd Design Automation Conference, pp. 527-532, 1996 
TABLE II

EXPERIMENTAL RESULTS.

\begin{tabular}{|c|cc|c|}
\hline App. & Const. $[\mu \mathrm{s}]$ & Area $\left[\mu \mathrm{m}^{2}\right]$ & Processor configuration \\
\hline \multirow{2}{*}{ FIR } & 60 & $1,531,347$ & $\begin{array}{r}\text { ALU16 } \times 1, \text { MUL16 } \times 3, \text { ADD32 } \times 2 \\
16 \text { bit heterogeneous registers } \times 7,32 \text { bit heterogeneous register } \times 1\end{array}$ \\
\cline { 2 - 5 } & 70 & 676,761 & $\begin{array}{r}\text { ALU16 } \times 1, \text { MUL16 } \times 1, \text { ADD32 } \times 1 \\
16 \text { bit heterogeneous registers } \times 5,32 \text { bit heterogeneous register } \times 2\end{array}$ \\
\hline \multirow{2}{*}{ DCT } & 60 & 656,057 & $\begin{array}{r}\text { ALU16 } \times 1, \text { ADD16 } \times 3, \text { MUL16 } \times 1, \text { ADD32 } \times 1 \\
16 \text { bit heterogeneous registers } \times 6,32 \text { bit heterogeneous register } \times 1\end{array}$ \\
\cline { 2 - 5 } & 80 & 580,290 & $\begin{array}{r}\text { ALU16 } \times 1, \text { MUL16 } \times 1, \text { ADD32 } \times 1 \\
16 \text { bit heterogeneous registers } \times 6,32 \text { bit heterogeneous register } \times 1\end{array}$ \\
\cline { 2 - 5 } & 110 & 568,038 & $\begin{array}{r}\text { ALU16 } \times 1, \text { MUL16 } \times 1, \text { ADD32 } \times 1 \\
16 \text { bit heterogeneous registers } \times 4,32 \text { bit heterogeneous register } \times 1\end{array}$ \\
\hline
\end{tabular}

TABLE III

RESULTS COMPARED TO EXISTING SYSTEMS.

\begin{tabular}{|c|c|c|c|c|c|c|c|}
\hline \multirow{2}{*}{\multicolumn{4}{|c|}{ FIR }} & \multicolumn{4}{|c|}{ DCT } \\
\hline & & & & \multirow{2}{*}{$\begin{array}{c}\text { Const. } \\
{[\mu \mathrm{s}]}\end{array}$} & \multicolumn{3}{|c|}{ Area $\left[\mu \mathrm{m}^{2}\right]$} \\
\hline \multirow{2}{*}{$\begin{array}{c}\text { Const. } \\
{[\mu \mathrm{s}]}\end{array}$} & \multicolumn{3}{|c|}{ Area $\left[\mu \mathrm{m}^{2}\right]$} & & Proposed & System 1 [15] & System 2 [16] \\
\hline & Proposed & System I [15] & System 2 [16] & 60 & 656,057 & 776,326 & 705,471 \\
\hline 80 & $1,531,341$ & - & 782412 & 80 & 580,290 & 720,323 & 649,468 \\
\hline 10 & $6 / 6, / 61$ & 800,326 & 152,443 & 110 & 568,038 & 693,918 & 643,340 \\
\hline
\end{tabular}

[5] S. Fröhlich and B. Wess, "Integrated approach to optimized code generation for heterogeneous-register architectures with multiple datamemory banks," in Proceedings of 14th Annual IEEE International ASIC/SoC Conference, pp. 122-126, 2001.

[6] N. Ishiura, M. Yamaguchi, and T. Kambe, "A graph-based algorithm of operation binding for compilers targeting heterogeneous datapath," in Proceedings of The 1998 IEEE Asia Pacific Conference on Circuits and Systems, pp. 395-398, 1998.

[7] N. Ishiura, T. Watanabe, and M. Yamaguchi, "A code generation method for datapath oriented application specific processor design," in Proceedings of SASIMI2000, pp. 71-78, 2000.

[8] N. Ishiura and T. Watanabe, "Datapath oriented codesign method of application specific DSPs using retargetable compiler," in Proceedings of 2002 Asia-Pacific Conference on Circuits and Systems, vol. 1, pp. $55-58,2002$.

[9] S. Kurohmaru, M. Matsuo, H. Nakajima, Y. Kohashi, T. Yonezawa, T. Moriiwa, M. Ohashi, M. Toujima, T. Nakamura, M. Hamada, T. Hashimoto, H. Fujimoto, Y. lizuka, J. Michiyama, and H. Komori, "A MPEG4 programmable codec DSP with an embedded pre/postprocessing engine," in Proceedings of the IEEE 1999 Custom Integrated Circuits, pp. 69-72, 1999

[10] Lucent Technologies, DSP/61//17/18/27/28/29 Digital Signal Processor Information Manual, 1998.

[11] P. Marwedel, "Code generation for core processor," in Proceedings of 34th Design Automation Conference, pp. 232-237, 1997.

[12] Motorola, DSP56300 24-bit Digital Signal Processor Family Manual (DSP56300FM/AD), 2000.

[13] Tensilica, Xtensa Microprocessor: Overview Handbook, http://www.tensilica.com.

[14] Texas Instruments, TMS320C2x Datasheet, 1998.

[15] N. Togawa, M. Yanagisawa, and T. Ohtsuki, "A hardware/software cosynthesis system for digital signal processor cores," IEICE Transactions on Fundamentals of Electronics, Communications and Computer Sciences, vol. E82-A, no. 11, pp. 2325-2337, 1999.
[16] N. Togawa, M. Yanagisawa, and T. Ohtsuki, "A hardware/software cosynthesis system for digital signal processor cores with two types of register files," IEICE Trans. on Fundamentals of Electronics, Communications and Computer Sciences, vol. E83-A, no. 3, 2000.

[17] J. Van Praet, D. Lanneer, G. Goossens, W. Geurts, and H. De Man, "A graph based processor model for retargetable code generation," in Proceedings of European Design and Test Conference, pp. 102-107, 1996.

[18] M. Yamaguchi, N. Ishiura, and T. Kambe, "A binding algorithm for retargetable compilation to non-orthogonal DSP architecture," IEICE Transactions on Fundamentals of Electronics, Communications and Computer Sciences, vol. E81-A, no. 12, 1998. 